MILITARY TECHNICAL COLLEGE CAIF? - EGY'PT

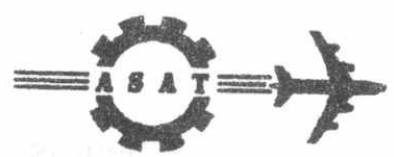

$7^{\text {th }}$ INTERNATIONAL CONF. ON AEROSPACE SCIENCES \& AVIATION TECHNOLOGY

\title{
THIN WALLED COMPOSITE BEAM STRUCTURE UNDER BENDING
}

\author{
M. Taha Tmerek*, A. A.Istafanous**, M. M. El-Nomrossy**, \\ E. E. El-Soaaly***
}

\section{ABSTRACT}

Although the analysis of beams made of metallic isotopic materials is well developed and documented, less information is available on the analysis of layered composite beams under bending. In the present work, a procedure was developed to analyze single cell closed section and open section made from an assembly of flat layered fibrous composite under symmetric bending. The analysis is based on symmetric laminates for each flat segment of a thin-walled section. For the validation of the presented analysis, a model was constructed with thin-walled open cross section of " $C$ " shape. The model was tested under bending moment and experimental strain measurements were performed. The results are compared with the theoretical analysis. The comparison shows that the presented approach gives results in a good agreement with the experimental measurements.

\section{KEY WORDS}

Thin-Walled Composite Beam, Bending, Box-Beam, Channel Beam .

\footnotetext{
${ }^{*}$ Graduate student, R\&D Center, EAF ${ }^{* *}$ Dpt. Of Aeronautics, M.T.C, Cairo, Egypt ${ }^{* * *}$ Professor, Dpt. Of
Mechanics \& Elasticity, M.T.C, Cairo, Egypt 


\section{INTRODUCTION}

The benefits of thin-walled composite beams with closed and open cross section have been widely recognized by the aerospace community. Many primary structural components such as helicopter blades and aircraft wing spars now feature composite beam designs. Compared to standard construction materials, composite materials present many advantages, e.g. light weight, corrosion resistance, and electromagnetic transparency. Most prominent is the property of tailoring the material for each particular application. Structural properties like stiffness, strength, and buckling resistance depend on the material system and the shape of the cross-section of the member. Like metal structural shapes, it is possible to optimize the section to increase the bending stiffness, without compromising the maximum bending strength. Unlike metal beams, in composite it is possible to optimize the material properties by choosing among a variety of resins, fiber systems, and fiber orientations. Changes in the geometry can be easily related to changes in the bending stiffness through the moment of inertia [1].

Although beams are the most commonly used structural elements, the theory of laminated beams has been less developed than the theory of laminated plates. Laminated beams theories were initially derived as extensions of existing plate or shell theories. Bert and Francis [2] presented a comprehensive review of the initial beam theories. Vinson and Sierakowski [3] applied classical lamination theory along with a plane strain assumption to obtain the extensional coupling and bending stiffness for an Euler-Bernoulli type laminated beam $\left(A_{11}, B_{11}, D_{11}\right)$.

Successful application of tailored composite beam structures requires the development and validation of new analytical tools which are both sufficiently accurate and computationally efficient. Recent efforts toward the creation of new analytical tools for composite beams have led to the development of several finite element based methods. The finite element models provide varying degrees of analytical flexibility depending on the level of computational effort. Detailed finite element formulations have been used to capture a variety of non-classical phenomenon in composite beams and blades [4], [5], [6].

A number of direct analytical methods have been formulated for thin-walled composite beams. Mansfield and Sobey [7] developed a simple thin-walled contour analysis and introduced the concept of the aeroelastically tailored 
composite helicopter blade. Using a simple composite beam model, Hong and Chorpa $[8,9]$ formulated comprehensive analysis for aeroelastic stability of advanced composite rotor systems. Bauchau has developed a thin-walled contour formulation using a refined approach to warping [10]. Bicos and Springer [11] investigated the minimum weight of a semimonocoque (stringers and webs) composite box-beam using a reduced plate model. Libove [12] have also developed a thin-walled contour analysis which is similar to the work of Mansfield and Sobey [7].

These direct analytical methods are based on combination of beam theory and classical lamination theory (CLT). Certain simplifying assumptions are made in order to obtain governing differential equations without discretizing the entire problem. In addition to computational simplicity and speed (relative to detailed finite element models), direct analytical formulations can provide valuable causeeffect relationships and enhanced physical understanding of non-classical phenomenon and elastic coupling effects. Direct analytical models are also very useful in evaluating the effects of composite design changes on overall system performance. Structural optimization is also an area where direct analytical formulations can be particularly appealing.

Despite the increased appearance of the aforementioned finite element and direct analytical methods during the past decade. The structural behavior of composite beams is not yet thoroughly understood. Some of the methods have not been fully developed and most of the methods have not been thoroughly validated for general composite designs. Validation is especially important for direct analytical methods since the major simplifying assumptions can effect the applicability limits of the model.

The objective of this work, is to illustrate how the analytical methods applicable to metal beams under bending can be extended to beams made of fiber-reinforced composites undergoing bending moment. The Euler-Bernoulli beam theory under bending is extended to beams with open and / or closed thin-walled sections made from flat layered laminated composites. In order to validate the obtained expressions for the stresses due to bending, illustrative example of a cantilever beam is presented; with an open channel section. The theoretical results are compared with strain measurements on a cantilevered beam model under bending. 


\section{THEORETICAL ANALYSIS}

The thin-walled sections under consideration are assumed to be made from an assembly of flat layered composite elements as shown in fig.1

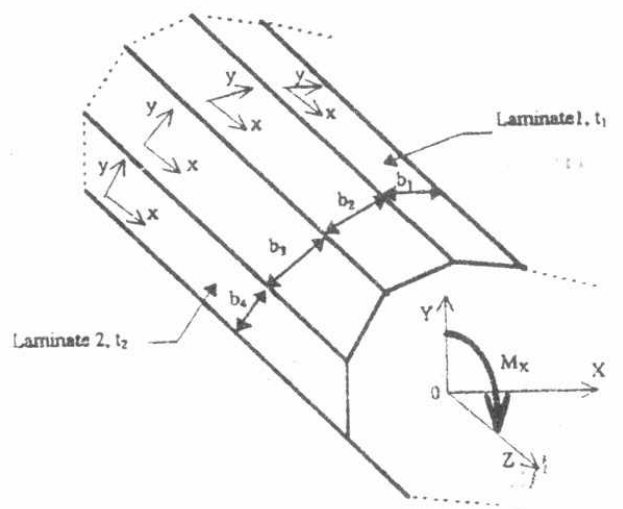

Fig.1. Symmetrical bending on $r$ composite section.

Two coordinate systems are used in the analysis :

1. A global right-hand orthogonal system of axes $\mathrm{XYZ}$, to define the beam geometry, section properties and the external loads are related to it. The origin $\mathrm{O}$ of this system is the section centroid while considering bending loads. The $\mathrm{Z}$-axis is in the direction of the beam axis and the $\mathrm{X}$ and $\mathrm{Y}$ axes are in the direction of centroidal principal axes of the section.

2. A local laminate reference axes system $(x-y)$ for each element in the section. Its $\mathrm{x}$-direction is taken to be parallel to the beam longitudinal direction $\mathrm{OZ}$.

For the analysis of the beams with thin-walled composite sections under bending load, it is assumed that the assumptions made in the bending analysis of beams made from isotopic materials are still valid. These assumptions are :

1. Plane sections before bending remain plane after bending.

2. The material obeys Hooke's law.

For sections made from laminated composites, the following additional assumptions are made : 
1. For each element of the section (parts like web and flange will be referred as an element), the composite ply is orthotropic, macroscopically homogeneous, linearly elastic (obeying Hooke's law ) and a plane stress condition exists.

2. The laminate of each element is symmetric about its mid plane. This would cause the decoupling between the membrane and bending effects ( coupling terms " $\mathrm{B}_{\mathrm{ij}}$ " are zero ).

3. The laminate of each element is specially orthotropic in the membrane mode; that is, there will be no shear coupling effects ( shear coupling terms A13 and A23 are zero ). Thus, a direct load on the element will not cause any shear deformations, and vice versa.

4. Each element is of a constant thickness, but the thickness can vary from element to element in a section.

5. The laminate configuration and the geometry of each element is such that the symmetry of the section is maintained, both in terms of geometry and material property.

The above assumptions are typical of laminate configurations and section properties found in practice. Symmetric laminate configurations are mostly used since they overcome the problem of warping due to thermal residual stresses. Moreover, in order to overcome the shear coupling effects in the membrane mode, all the generally orthotropic plies are used in pairs of alternating signs, hence the assumption of special orthotropy in the membrane mode is a realistic one. Finally, structural sections are mostly of the properties such that the section symmetry (or antisymmetry) is maintained, in order to avoid any coupling problems between different modes of loads and deformations. Thus, the assumption of section symmetry, both in terms of geometry and material property is also a practical one [13] .

According to the engineers' theory of bending, the normal stress in the axial direction ( beam Z-direction or element $\mathrm{x}$-direction ) at any point on a crosssection, due to symmetrical bending moment " $\mathrm{Mx}$ " around the $\mathrm{X}$-axis, is given by the expression:

$$
\sigma_{\mathrm{Z}}=\frac{\mathbf{E}_{\mathbf{Z}} \mathbf{Y}}{\mathbf{R}}
$$

where :

$\mathrm{R}$ is the radius of curvature of the beam in the deformed state. 
$\mathrm{E}_{\mathrm{Z}}$ is the membrane equivalent Young's modulus of the laminate in the Z-direction.

$Y \quad$ is the ordinate from the neutral plane of the section to the point of the element in question.

The total applied moment " $\mathrm{M}_{\mathrm{X}}$ " is obtained by the expression:

$$
\mathrm{M}_{\mathrm{X}}=\int_{\mathbf{A}} \sigma_{\mathrm{Z}} \mathbf{Y d A}
$$

where the integration is performed over the whole sectional area. Substituting for the normal stress " $\sigma_{\mathbf{Z}}$ " from equation (1), in the above expression, we get:

$$
M_{X}=\int_{A} \frac{E_{Z} Y^{2}}{R} d A
$$

In the case of composite section, the laminate configuration may change from element to element in a section, thereby each element may have a different membrane equivalent value for the Young's modulus " $E_{Z}$ ", therefore the " $E_{Z}$ " value cannot be taken out of the integral domain. Based on the assumption of plane sections, the radius of curvature is common to all the elements in the section and can, therefore, be excluded from the integral domain. Equation (3), therefore, becomes

$$
M_{X}=\frac{1}{R} \int_{A} E_{Z} Y^{2} d A
$$

In order to obtain expression for the normal stress due to bending similar to that of the engineer's theory of bending, it is necessary to define a new term for the second moment of area, "I xx", which will include the membrane equivalent Young's modulus value for each element in the form: [13]

$$
I_{X X}=\int_{A} E_{Z} Y^{2} d A=\sum_{i=1}^{n}\left(E_{Z} I_{X X}\right)_{i}
$$

where

$\mathrm{n}$ is the number of elements in the section

$\mathrm{I}_{\mathrm{XX}}$ is the area moment of inertia for each element in the section 
Hence, equation (4) becomes

$$
\mathrm{M}_{\dot{\mathrm{X}}}=\frac{\mathrm{I}_{\mathrm{XX}}}{\mathrm{R}}
$$

From equation (1) and (6), the expression for the normal stress due to bending moment " $\mathrm{MX}$ " becomes:

$$
\sigma_{\mathrm{Z}}=\frac{E_{Z} M_{X} Y}{I_{X X}}
$$

For the case of a bending moment " $\mathrm{MY}_{\mathrm{Y}}$ " induced about the $\mathrm{Y}$-axis, equation (7) is, therefore, modified to become

$$
\sigma_{Z}=\frac{E_{Z} M_{Y} X}{\Gamma_{Y Y}}
$$

where

$$
I_{Y Y}=\sum_{i=1}^{n}\left(E_{Z} I_{Y Y}\right)_{i}
$$

$\mathrm{X}$ is the $\mathrm{X}$-ordinate value to the point in question.

For the combined case when the two bending moments " $\mathrm{M}_{\mathrm{X}}$ " and " $\mathrm{M}_{\mathrm{Y}}$ " are acting together, then the resultant normal stress distribution will be the summation of the two stress values:

$$
\sigma_{Z}=E_{Z}\left(\frac{M_{X} Y}{I_{X X}}+\frac{M_{Y} X}{I_{Y Y}}\right)
$$

The normal stress, as given by equations (7), (8) and (9), is the 'average' stress value acting through the thickness of an element of a particular laminate lay-up. If the element (laminate) is of a total thickness " $t$ ", then the corresponding force intensity is

$$
\mathrm{N}_{\mathrm{Z}}=\sigma_{\mathrm{Z}}{ }^{* \mathrm{t}}
$$

But, since the Z-direction is parallel to all the laminate $x$-directions, therefore, $N_{Z}$ $=\mathrm{N}_{\mathrm{x}}$ and $\sigma_{\mathrm{Z}}=\sigma_{\mathrm{z}}$ for any element in the section, then 


$$
\mathrm{N}_{\mathrm{x}}=\sigma_{\mathrm{Z}}{ }^{*} \mathrm{t}=\sigma_{\mathrm{x}}^{*} \mathrm{t}
$$

Having got the force intensity on a laminate, the ply-to-ply stress can be done using the classical lamination theory and the strength analyses can be performed using any strength theory.

The expressions for the normal stress distribution, given by equations (7), (8) and (9), are applicable to both open and closed sections under bending.

\section{EXPERIMENTAL WORK AND VALIDATION OF ANALYSIS}

In order to validate the approach presented in the previous section, a test program was conducted. A thin-walled beam model was constructed with a channel cross-section. The model is fixed as cantilever by means of a metal plate. The metal plate has four welded fingers each one is $200 \mathrm{~mm}$ long to hold the model. The fingers is fixed to the model by wrapping fibers and resin. The plate is fixed to the wall through four bolts each is $10 \mathrm{~mm}$ diameter. The basic dimensions of the model is shown in fig.2. Strain gages were installed at 4 stations at $253,425,601$ and $733 \mathrm{~mm}$ from the free end.

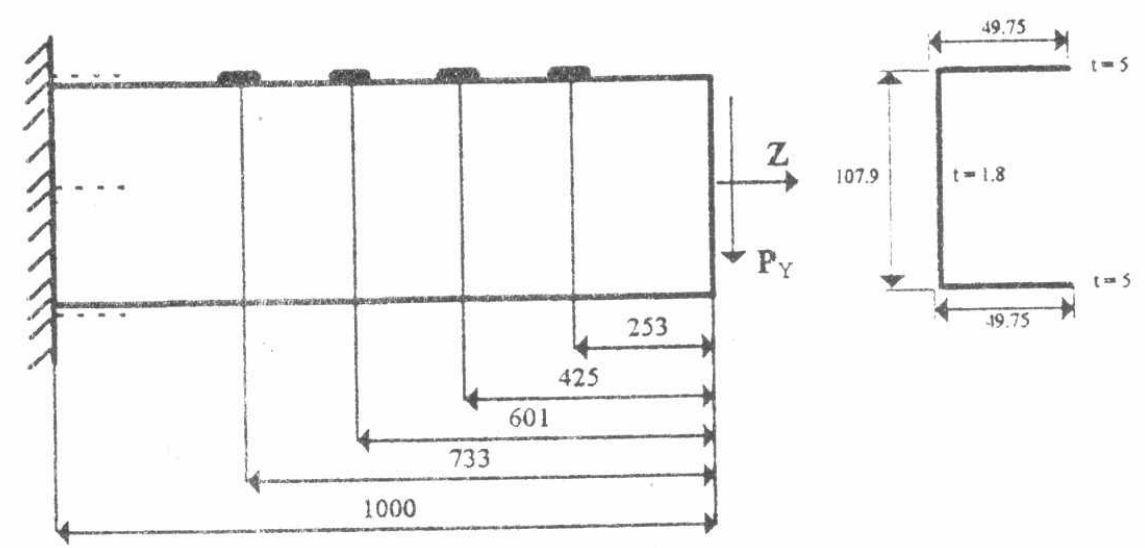

Fig. 2. Gages position and Cross section dimensions ( $\mathrm{mm}$ )

The material employed is fiberglass 7781 with polyester resin Neoxel 886 Ta-12. Two laminates configurations were used:

-for the flanges: $\left[(45 /-45)_{2} /(0 / 90)_{5}\right]_{s}$.

-for the web : $\left[(45 /-45)_{2}\right]_{s}$. 
The equivalent mechanical properties of both laminates were obtained from coupons testing according to the ASTM D 3039. Three coupons was constructed for each laminate and each coupon was tested six times. Thus, reliable data are generated. The membrane equivalent Young's modulus for both laminates are :

- for flange laminate:

- for web laminate:

$$
\mathrm{E}_{\mathrm{z}}=0.022869 * 10^{6} \quad\left(\mathrm{~N} / \mathrm{mm}^{2}\right)
$$

$$
\mathrm{E}_{\mathrm{z}}=0.009513 * 10^{6} \quad\left(\mathrm{~N} / \mathrm{mm}^{2}\right)
$$

The model is subjected to tip loads $(98.07,196.14,294.21,392.28$ and 490.35 $\mathrm{N}$ ). In order to obtain bending without twist, the applied shear force is acting into the center of shear of the composite cross-section. The experimental results and the corresponding strain calculated according to the presented approach and using the values of the membrane equivalent Young's modulus obtained from coupon test are listed in table 1. In fig.3, the relationship between " $p$ " and " $\varepsilon$ " shows linear elastic behavior for the composite material used to construct the model over strain range up to 600 microstrain. In fig. 4-8, the experimental and calculated results are presented at the four stations for the applied loads. The experimental results confirm reasonably with the calculated results.

Table 1 Calculated and Measured Strain (micro strain)

\begin{tabular}{|c|c|c|c|c|c|c|c|c|}
\hline Station & \multicolumn{2}{|c|}{$253 \mathrm{~mm}$} & \multicolumn{2}{c|}{$425 \mathrm{~mm}$} & \multicolumn{2}{c|}{$601 \mathrm{~mm}$} & \multicolumn{2}{|c|}{$733 \mathrm{~mm}$} \\
\hline Load N & $\mathrm{C}$ & $\mathrm{M}$ & $\mathrm{C}$ & $\mathrm{M}$ & $\mathrm{C}$ & $\mathrm{M}$ & $\mathrm{C}$ & $\mathrm{M}$ \\
\hline 98.07 & 38.3 & 27 & 64.4 & 70 & 911 & 88 & 111 & 105 \\
\hline 196.14 & 76.6 & 74 & 128.7 & 115 & 182.1 & 205 & 222 & 221 \\
\hline 294.21 & 114.9 & 122 & 193.1 & 210 & 273 & 323 & 333 & 338 \\
\hline 392.28 & 153.2 & 169 & 257.4 & 280 & 364 & 441 & 444 & 455 \\
\hline 490.35 & 191.6 & 217 & 321.8 & 351 & 455 & 558 & 555 & 571 \\
\hline
\end{tabular}

C : Calculated $\quad$ M : Measured 


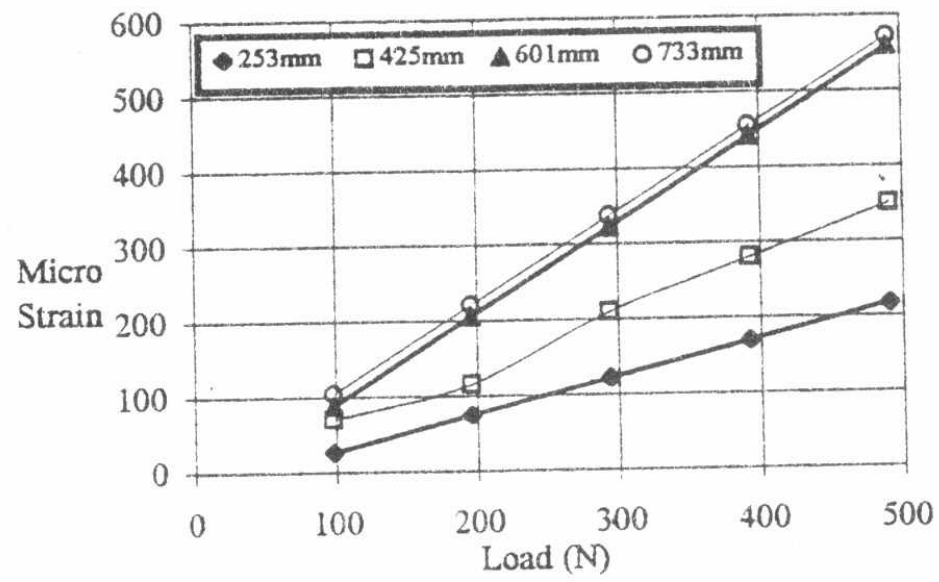

Fig.3. Strain Measurements at 4 stations

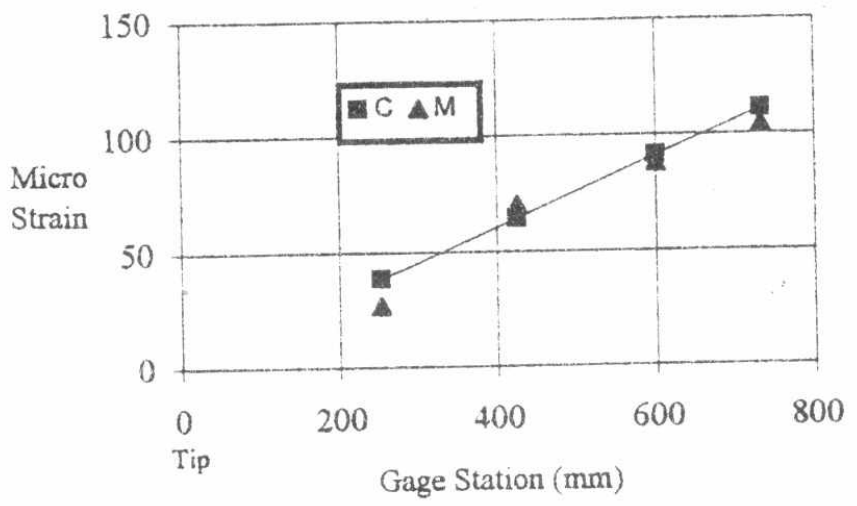

Fig.4 Calculated and Measured Strain for $P=98.07 \mathrm{~N}$

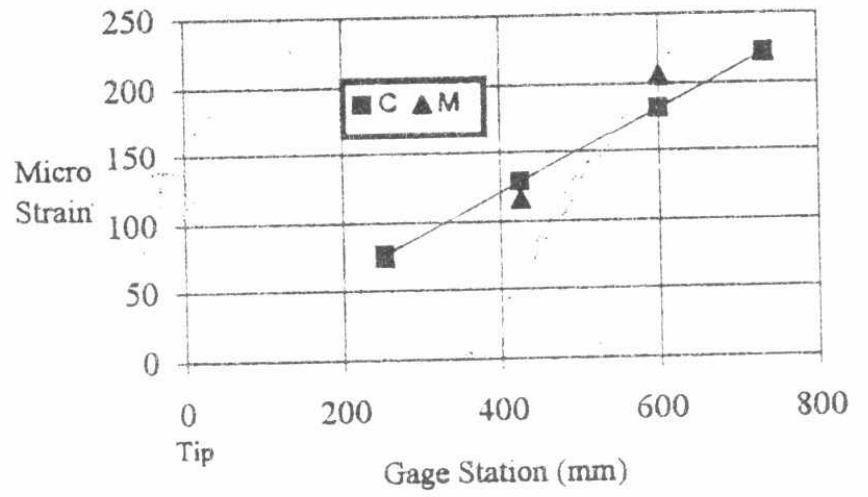

Fig.5 Calculated and Measured Strain for $P=196.14 \mathrm{~N}$ 


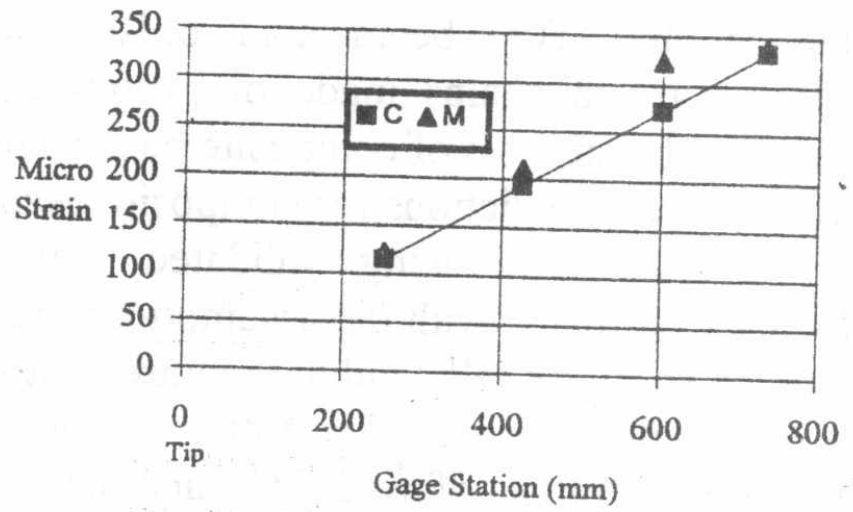

Fig.6 Calculated and Measured Strain for $P=294.21 \mathrm{~N}$

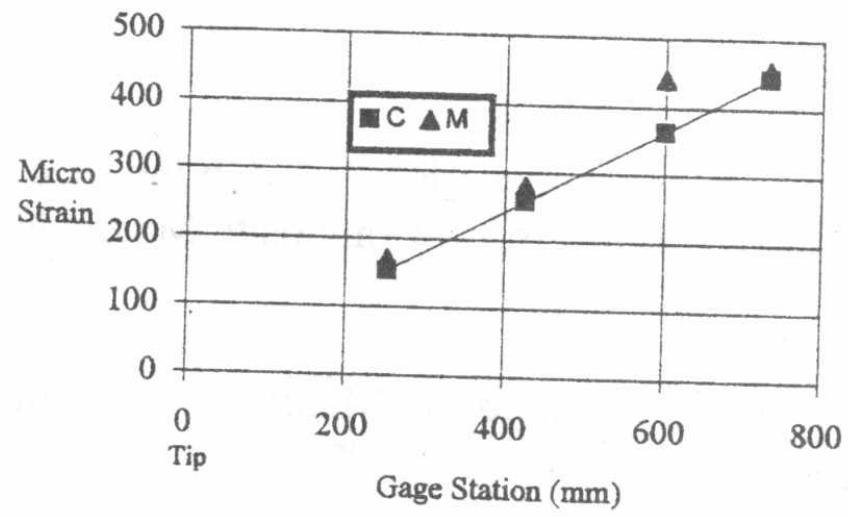

Fig. 7 Calculated and Measured Strain for $P=392.28 \mathrm{~N}$

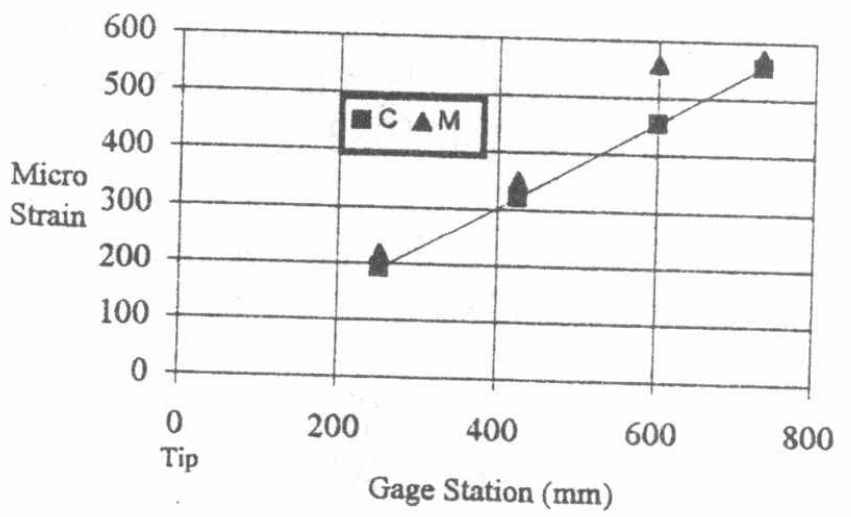

Fig.8 Calculated and Measured Strain for $P=490.35 \mathrm{~N}$ 


\section{CONCLUSION}

The above results indicate that the ETB for beams made of isotropic material can be extended with modification to beams made of fibrous composite. The presented simple approach for the analysis of composite beams under bending is still useful and gives accurate stress predictions for composite beam with open or closed cross-section (currently, it is being validated experimentally for rectangular cross-section). This approach, with the assumptions made, bridges the gap between sophisticated existing models and the requirement of a simple but consistent tool for the preliminary design. Currently, composite blades, for instance, are built with the assumption of symmetric and balanced laminate to completely eliminate the elastic couplings. In further research, some of the assumptions imposed can be released to obtain more accurate results.

\section{REFERENCES}

1. Davalos, J. F., Barbero, E. J., Lopez, R. -A., " On the Mechanics of ThinWalled Laminated Composite Beams," J. Composite Materials, Vol.. 27, No. 8, 1993.

2. Bert, C. W., Francis, P. H., "Composite Material Mechanics : Structural Mechanics," AIAA Journal, Vol. 12, No. 9, 1974.

3. Vinson, J. R. and Sierakowski, R., L., The behavior of Structures Composed of Composite Materials, Dordrecht: Martinus Nijhoff Publishers, 1987

4. Giavotto, M. Borri, P. Mantegazza, G. Ghiringhelli, V. Carmaschi, G. C. Maffioli and F. Mussi, "Anisotropic Beam Theory and Application," Computers \& Structures, Vol. 16, No. 1/4, 1983.

5. Bauchau, O. A., Hong, C. H., "Nonlinear Composite Beam Theory," J. of Applied Mechanics, Vol. 55, March 1988.

6. Lee, S. W., Stemple, A. D., "Finite-Element Model for Composite Beams with Arbitrary Cross-Sectional Warping," AIAA Journal, Vol. 26, No. 12, 1988. 
7. Mansfield, E. H., Soby, A. J., “ The Fiber Composite Helicopter Blade, Part 1 Stiffness Properties, Part 2: Prospects for Aeroelastic Tailoring," Aeronautical Quarterly, Vol. 30, No. 2, 1979.

8. Chopra, I., Hong, C., -H., " Aeroelastic Stability Analysis of a Composite Rotor Blade," J. of the American Helicopter Society, April 1985.

9. Chopra, I., Hong, C., -H., “ Aeroelastic Stability Analysis of a Composite Bearingless Rotor Blade," J. of the American Helicopter Society, October 1986.

10.Bauchau, O. A., "A Beam Theory for Anisotropic Materials," J. of Applied Mechanics, Vol. 52, June 1985.

11.Bicos, A. S., Springer, G. S., " Design of a Composite Boxbeam," J. Composite Materials, Vol. 20, No. 1, 1986.

12.Libove, C., " Stresses and Rate of Twist in Single- Cell Thin-Walled Beams with Anisotropic Walls," AIAA Journal, Vol. 26, No. 9, 1988.

13.Datoo, M., H., Mechanics of Fibrous Composites, Elsevier Applied Science, London and New York, 1991. 